\title{
Use of Evidence-Based Practices in Pregnancy and Childbirth: South East Asia Optimising Reproductive and Child Health in Developing Countries Project
}

\author{
The SEA-ORCHID Study Group*
}

\begin{abstract}
Background: The burden of mortality and morbidity related to pregnancy and childbirth remains concentrated in developing countries. SEA-ORCHID (South East Asia Optimising Reproductive and Child Health In Developing countries) is evaluating whether a multifaceted intervention to strengthen capacity for research synthesis, evidence-based care and knowledge implementation improves adoption of best clinical practice recommendations leading to better health for mothers and babies. In this study we assessed current practices in perinatal health care in four South East Asian countries and determined whether they were aligned with best practice recommendations.

Methodology/Principal Findings: We completed an audit of 9550 medical records of women and their 9665 infants at nine hospitals; two in each of Indonesia, Malaysia and The Philippines, and three in Thailand between JanuaryDecember 2005. We compared actual clinical practices with best practice recommendations selected from the Cochrane Library and the World Health Organization Reproductive Health Library. Evidence-based components of the active management of the third stage of labour and appropriately treating eclampsia with magnesium sulphate were universally practiced in all hospitals. Appropriate antibiotic prophylaxis for caesarean section, a beneficial form of care, was practiced in less than $5 \%$ of cases in most hospitals. Use of the unnecessary practices of enema in labour ranged from $1 \%$ to $61 \%$ and rates of episiotomy for vaginal birth ranged from $31 \%$ to $95 \%$. Other appropriate practices were commonly performed to varying degrees between countries and also between hospitals within the same country.

Conclusions/Significance: Whilst some perinatal health care practices audited were consistent with best available evidence, several were not. We conclude that recording of clinical practices should be an essential step to improve quality of care. Based on these findings, the SEA-ORCHID project team has been developing and implementing interventions aimed at increasing compliance with evidence-based clinical practice recommendations to improve perinatal practice in South East Asia.
\end{abstract}

Citation: The SEA-ORCHID Study Group (2008) Use of Evidence-Based Practices in Pregnancy and Childbirth: South East Asia Opptimising Reproductive and Child Health in Developing Countries Project. PLoS ONE 3(7): e2646. doi:10.1371/journal.pone.0002646

Editor: Hany Abdel-Aleem, Assiut University Hospital, Egypt

Received February 19, 2008; Accepted June 8, 2008; Published July 9, 2008

Copyright: (c) 2008 Crowther et al. This is an open-access article distributed under the terms of the Creative Commons Attribution License, which permits unrestricted use, distribution, and reproduction in any medium, provided the original author and source are credited.

Funding: This study is jointly funded by an International Collaborative Research Grant from the National Health and Medical Research Council of Australia (No. 307703) and Wellcome Trust, United Kingdom (071672/Z/03/Z). The funders had no role in study design, data collection and analysis, decision to publish, or preparation of the manuscript.

Competing Interests: The authors have declared that no competing interests exist.

* E-mail: caroline.crowther@adelaide.edu.au

\section{Introduction}

The burden of mortality and morbidity related to pregnancy and childbirth remains concentrated in developing countries $[1,2]$. This disparity continues with rates of neonatal mortality almost 10 times greater in South East Asia than developed regions [3,4]. SEA-ORCHID, a five-year project, is evaluating whether a multifaceted intervention to strengthen capacity for research synthesis, evidence-based care and knowledge implementation improves adoption of best clinical practice recommendations and so leads to better health for mothers and babies [5]. This paper describes empirical evidence of current practices at the beginning of the SEA-ORCHID project for key aspects of maternal and perinatal health care in the participating countries.

\section{Methods}

Setting

This audit was conducted in nine hospitals across Indonesia, Malaysia, The Philippines and Thailand, with support from three sites in Australia. Different types of hospital were represented including tertiary referral hospitals (University and regional), provincial hospitals and district hospitals. All were selected as part of the SEA-ORCHID project.

Seven of the hospitals were tertiary referral institutions with regional referrals of women with a high risk pregnancy. Two hospitals were provincial or district institutions. Models of delivery care included a multidisciplinary approach with midwives (including nurses with midwifery qualifications) or obstetric specialists. All hospitals had obstetric specialists and caesarean 
section facilities available. Normal vaginal births were conducted by doctors and/or midwives (including nurses with midwifery qualifications) in all hospitals.

The SEA-ORCHID project settings and methods have been published elsewhere. [5]. The project was approved by the local ethics committee of each hospital and by the ethics committee of the administering institution in Australia (University of Sydney).

\section{Procedure}

We reviewed the medical records of 9550 women 9665 infants including 111 twins and two sets of triplets) admitted to the labour wards at the nine study hospitals between January-December 2005. The duration of data collection varied according to the number of births per month at the participating hospital. Five hospitals in the sample collected data on a consecutive basis until reaching a total of at least 1000 women. To avoid potential biases associated with short data collection periods, the four largest hospitals sampled cases using various ratios to ensure there was at least a three-month minimum data collection period at each hospital.

Staff at each hospital were trained to prospectively audit medical records using pre-established data extraction forms. We extracted information about current maternal and perinatal practice according to beneficial forms of care and forms of care likely to be ineffective or harmful as suggested in the World Health Organization Reproductive Health Library No.7 [6] and the Cochrane Library [7] (Table 1). The primary caregiver at the birth provided details of the presence of maternal companionship during the labour.

In addition we collected information on maternal characteristics including age, parity, height and weight, and on birth outcomes including gestational age at birth, mode of birth, birth weight, Apgar scores and perinatal mortality.
Completed data extraction forms were sent from the hospitals to the project co-ordinating site in each country for manual data entry by the trained fieldworkers using a secure web-based database. The online form was set-up to minimise transcription errors by performing validation checks to detect discrepancies and missing data. A random sample of between 5 to $10 \%$ was independently checked by project staff at one of the Australian support sites to identify data processing errors.

\section{Data analysis}

Descriptive analyses were performed across countries and between hospitals within countries. We used STATA software version 8.0 for data analysis [8]. We used frequencies to describe maternal characteristics, maternal and perinatal practices, as well as birth outcomes measured as categorical data. We used means and standard deviations to describe the continuous data variables of maternal age and gestational age at birth.

\section{Results}

Maternal characteristics and birth outcomes (Table 2)

For the 9550 mothers (and their 9665 babies) across the nine hospitals in South East Asia, the mean age per hospital ranged from 26 to 31 years. The rate of nulliparity varied, being over $40 \%$ in all hospitals of Indonesia, The Philippines and Thailand, and up to $64 \%$ in one Thai hospital but only $27 \%$ for one Malaysian hospital.

For the 9665 babies, the preterm birth rate $(<37$ weeks gestation) ranged from $6 \%$ to $15 \%$. Overall $30 \%$ of all babies were born by caesarean section but the rate varied considerably across the nine hospitals ranging from $12 \%$ to $39 \%$. Around a fifth of babies were born by caesarean section in the Malaysian hospitals and over a third in the Thai hospitals. Overall, 18\% of babies were of low birth weight $(<2500 \mathrm{~g})$ with rates ranging from $9 \%$ to $20 \%$.

Table 1. Recommended practices in maternal and perinatal health care

\begin{tabular}{|c|c|c|}
\hline Recommended practice & Care practices assessed & Outcome intended to reduce \\
\hline \multicolumn{3}{|l|}{ Beneficial forms of care } \\
\hline $\begin{array}{l}\text { Antibiotics for preterm prelabour rupture of membranes } \\
\text { (pPROM) [15] }\end{array}$ & $\begin{array}{l}\text { Use of antibiotics in women with preterm ( }<37 \text { weeks) } \\
\text { prelabour rupture of membranes }\end{array}$ & Chorioamnionitis; neonatal sepsis \\
\hline Corticosteroids prior to preterm birth [16] & $\begin{array}{l}\text { Use of antenatal corticosteroids in women at risk of } \\
\text { preterm birth at }<34 \text { weeks gestation }\end{array}$ & $\begin{array}{l}\text { Neonatal death; complications of } \\
\text { preterm birth }\end{array}$ \\
\hline Continuous support during labour [17] & Family member present with the woman during childbirth & Caesarean section rate \\
\hline $\begin{array}{l}\text { Magnesium sulphate for eclampsia and } \\
\text { pre-eclampsia }[18,19,20]\end{array}$ & $\begin{array}{l}\text { Use of magnesium sulphate for women with eclampsia } \\
\text { and pre-eclampsia }\end{array}$ & Maternal death; eclampsia \\
\hline Management of third stage of labour [21] & $\begin{array}{l}\text { Use of uterotonic and controlled cord traction in the third } \\
\text { stage of labour }\end{array}$ & $\begin{array}{l}\text { Postpartum haemorrhage; maternal } \\
\text { death }\end{array}$ \\
\hline \multicolumn{3}{|l|}{$\begin{array}{l}\text { - appropriate administration of a prophylactic oxytocic } \\
\text { at or after birth of the baby }\end{array}$} \\
\hline \multicolumn{3}{|l|}{ - controlled umbilical cord traction to deliver the placenta } \\
\hline $\begin{array}{l}\text { Intraoperative antibiotics during caesarean } \\
\text { section [14] }\end{array}$ & $\begin{array}{l}\text { Administration of a single dose of ampicillin or first } \\
\text { generation cephalosporin after umbilical cord clamping } \\
\text { at caesarean section }\end{array}$ & Maternal infection \\
\hline Immunisation for Hepatitis B [22] & Vaccination of babies against Hepatitis B virus & Hepatitis B infection \\
\hline \multicolumn{3}{|l|}{ Forms of care likely to be unnecessary or harmful } \\
\hline Routine episiotomy [23] & Restrictive use of episiotomy & Perineal injury; maternal infection \\
\hline Routine shaving* $[24]$ & Avoidance of pubic hair shaving & Maternal infection \\
\hline Routine enemas* [25] & Avoidance of use of enemas during labour & Maternal infection \\
\hline
\end{tabular}

*No clear evidence from Cochrane reviews to support or refute use, but identified as practices of importance to research and evaluate

doi:10.1371/journal.pone.0002646.t001 


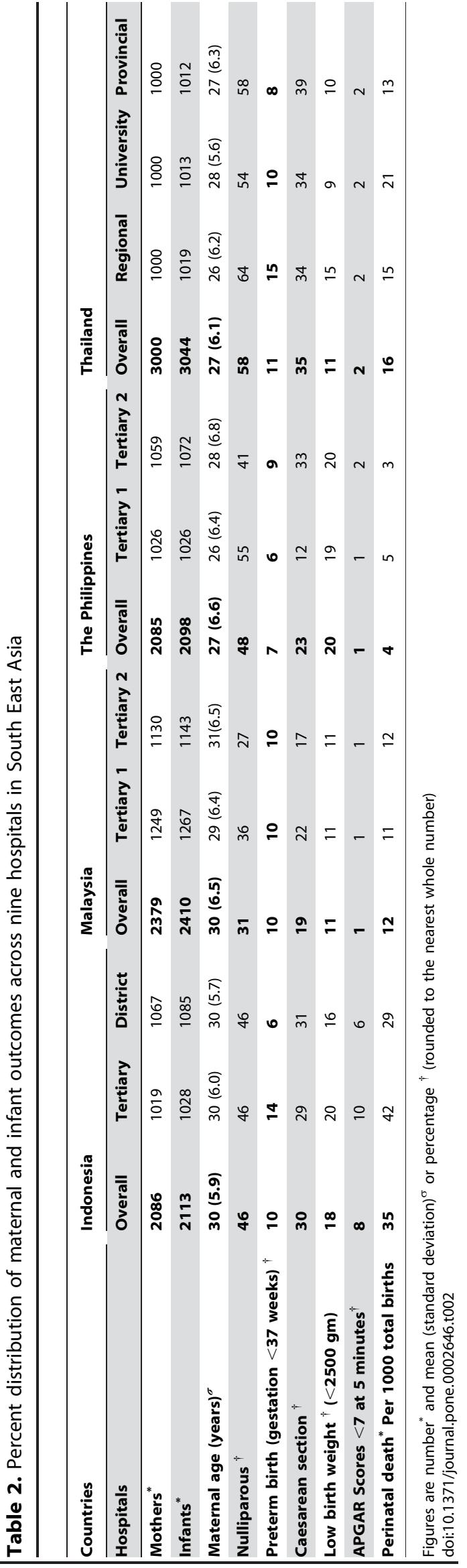

Rates of babies with Apgar scores less than seven at five minutes and perinatal death were highest in the two Indonesian hospitals, compared with rates in the other hospitals. Stillbirths accounted for more than $60 \%$ of the perinatal deaths in most hospitals across the countries. There were no maternal deaths prior to discharge reported in any of the hospitals during the audit.

The variation in rates between countries and between hospitals within the same country are likely to be influenced by the sociodemographics and risk status of the populations they care for, and the type of hospital (tertiary, provincial or district).

\section{Beneficial forms of care}

The recommended beneficial clinical practices were classified according to periods of pregnancy care: antenatal, intrapartum and postpartum (Table 3). The use of these clinical practices varied markedly across the nine hospitals.

Care practices during the antenatal period to treat pregnancy complications (Table 3). Women with preeclampsia represented $4 \%$ of the sample; with nearly threequarters of the women with pre-eclampsia receiving magnesium sulphate. The rate of practice of this intervention varied among the hospitals within Malaysia (range 13\% to $44 \%$ ) and Thailand (range $57 \%$ to $82 \%$ ) but was $100 \%$ in both Indonesian hospitals. There were $15(0.16 \%)$ cases of eclampsia. Magnesium sulphate was used in all $15(100 \%)$ of these cases of eclampsia, and in all countries involved in the audit.

Women with preterm prelabour rupture of the membranes (pPROM) received antibiotics $80 \%$ of the time. Although this practice was observed in all hospitals, the rates of administration varied across countries (range $62 \%$ to $92 \%$ ) and hospitals (range $44 \%$ to $100 \%$ ). In Indonesia, use was appropriately high whereas variation of the practices was seen among hospitals within each country.

Antenatal corticosteroid administration to women with preterm birth at $<34$ weeks was infrequently used in most hospitals compared with other clinical practices in the antenatal period. The highest rate of antenatal corticosteroid use was $86 \%$ in a regional hospital in Thailand. In the Malaysian hospitals about half the women who gave birth at $<34$ weeks had been given antenatal corticosteroids. In the Indonesian hospitals this dropped to around $10 \%$ and in one tertiary hospital in The Philippines no administration of corticosteroids was recorded. Use of repeat courses of antenatal corticosteroids was less than $15 \%$ across all hospitals included in the audit.

Gare practices during the intrapartum period (Table 3). Companionship in labour was assessed as to whether the woman had a family member present during childbirth. Rates for family support during labour varied widely, ranging from $<1 \%$ at a Philippine hospital to $86 \%$ at a Malaysian hospital. Among the Malaysian hospitals the rates differed from $32 \%$ to $86 \%$. In Thailand the rates of family support during labour were $31 \%$ at the university hospital, $44 \%$ at the provincial hospital and $83 \%$ at the regional hospital.

There were wide variations in the administration of an appropriate prophylactic oxytocic during third stage of labour both between countries and within countries. Rates for the administration of a uterotonic varied from $<1 \%$ at one Philippine hospital to $98 \%$ at the Thai provincial hospital. Among the Thai hospitals rates of the practice were $4 \%, 76 \%$ and $98 \%$. The variation was similar in The Philippines with one hospital giving oxytocics in two-thirds of cases and $<1 \%$ in the other.

Controlled umbilical cord traction to deliver the placenta was routinely performed in the Indonesian, Malaysian and Philippine 


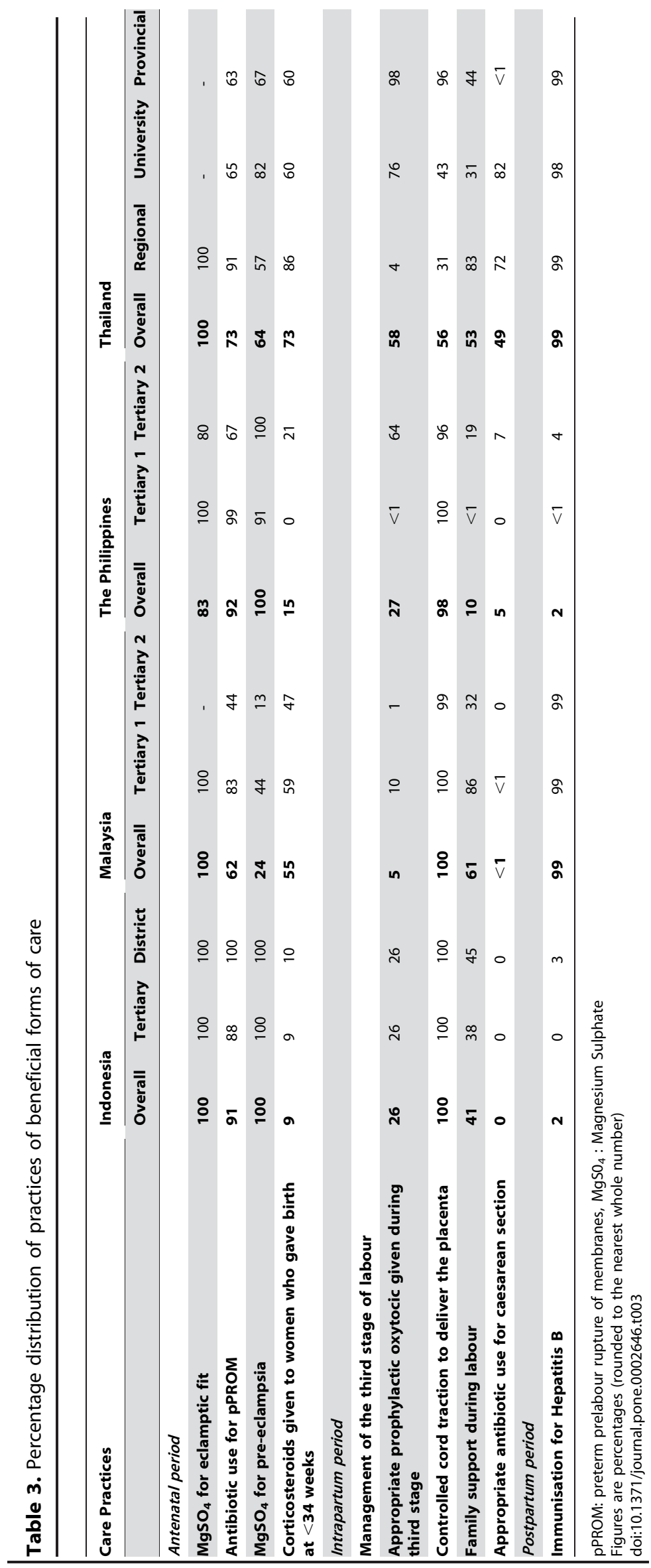


hospitals. In Thailand, the rates were below $50 \%$ in the regional and tertiary hospitals but $96 \%$ in the provincial hospital.

Use of antibiotic prophylaxis for caesarean section in a single does administered after clamping of the umbilical cord was mostly very low or non-existent across all the hospitals. About a quarter of women who gave birth by caesarean were given appropriate antibiotic prophylaxis (534 out of 2564), over 95\% of these women were from two Thai hospitals.

Although antibiotics were often given in other hospitals, the timing of administration was preoperatively or postoperatively, and multiple doses were often prescribed.

Practices during postpartum period (Table 3). Immunising babies against the hepatitis B virus was the only postpartum neonatal care practice assessed in our project. This intervention was only routinely practiced in the Malaysian and Thai hospitals.

Forms of care likely to be ineffective or harmful (Table 4)

Episiotomy was the form of care likely to be harmful which was most frequently practiced across all the hospitals and in all four countries. The overall episiotomy rate for women giving birth vaginally was $65 \%$. Episiotomies were liberally performed in all three hospitals in Thailand, and in only one Malaysian hospital did the rate drop below one-third. Pubic hair shaving, another ineffective and potentially harmful form of care, was commonly practiced in all countries with rates as high as $98 \%$ in one Thai hospital. In only one Philippine hospital was this observed as an occasional practice. Use of enemas during labour, an ineffective practice, was occasionally used in four hospitals across all countries and was highest in one Thai $(61 \%)$ and one Malaysian $(44 \%)$ hospital.

\section{Discussion}

This study describes the rates of use of selected practices of key aspects of perinatal care reported in the medical records of nine hospitals in Indonesia, Malaysia, The Philippines and Thailand. The findings show high rates of compliance for some evidencebased recommendations for perinatal care and wide divergence for others. Practices that were in line with recommendations across most hospitals for the beneficial forms of care were controlled cord traction, one of the components of the active management of the third stage of labour, and treating eclampsia with magnesium sulphate. The unnecessary practice of enema use was appropriately widely avoided. The highest level of divergence from best practice recommendations in most countries was not administering appropriate antibiotic prophylaxis for caesarean section. Liberal use of episiotomy for women having a vaginal birth is not recommended but was often inappropriately practiced across the hospitals in all four countries, demonstrating lack of adoption of the evidence-based recommendation of restrictive episiotomy. Other forms of perinatal care such as pubic hair shaving and the use of enemas during labour varied in rates of compliance across all countries and even between hospitals within the same country.

The principles of evidence-based practice are to encourage health professionals to use practices with proven benefit and eliminate the use of those shown to be ineffective or harmful. Effective implementation of beneficial practices in developing regions $[9,10]$, such as South East Asia, should lead to a reduction in maternal and neonatal mortality and morbidities.

Our findings are consistent with three previous reports of perinatal practice from the Asian and Arab world [11-13]. The first was reported by the Choices and Challenges in Changing Childbirth Research Network [11]. The network documented routine obstetric practices for normal labour and birth in Egypt,

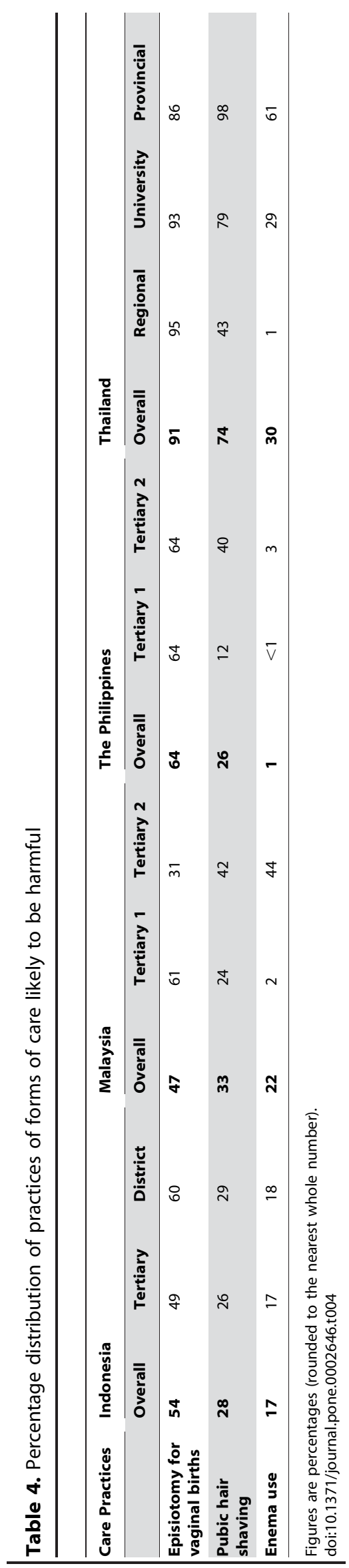


Lebanon, Syria and the West Bank, and compared these with evidence-based recommendations. They showed the practices for normal labour were largely not in accordance with the World Health Organization evidence-based classification of practices for normal birth. The second report described facility-based practices for normal labour and birth [12]. Forty-four clinical practices observed in a busy Egyptian teaching hospital were categorised according to World Health Organization Technical Working Group on normal birth classification of normal birth practices. This study concluded that practices for normal labour were largely not consistent with the World Health Organization evidencebased classification of practices for normal births. The third report compared practices of selected childbirth care procedures against evidence-based information and explored user and provider views about each procedure in four hospitals in Shanghai, China [13]. They concluded that obstetric practices of the hospitals studied were not following best available evidence.

There is clear evidence of benefit to perinatal health outcomes with use of appropriate antibiotic prophylaxis for caesarean section, use of antenatal corticosteroids for women at risk of preterm birth and family support during labour. Our findings however, show that these clinical practices were rarely performed in most of the included hospitals, with high rates of variation across the countries. It is likely that there are a range of barriers to all these clinical practices in our study settings, but detailed exploration of these was outside the scope of this initial audit. Although we were not able to interview the care providers or directly observe the clinical practices at the time of this survey, these were planned for a later stage of this project.

Recommendation of use of appropriate antibiotic prophylaxis for caesarean section is defined as administration of a single dose of ampicillin or first generation cephalosporin after umbilical cord clamping of the baby [14]. We found that most non-concordant practices relating to antibiotic prophylaxis for caesarean section arose from giving multiple doses or administering antibiotics pre or post operatively. In some clinical settings multiple doses of antibiotics at pre-operative or post-operative times may be appropriate.

One advantage of our study was that the prospective, sequential collection of data provided accurate information of individual pregnant women and their babies, directly extracted from the medical records of individual pregnant women rather than derived from interviewing health care providers. In addition, the information was extracted prospectively and therefore the findings closely reflect the actual practices of perinatal health care in the nine hospitals of the four South East Asian countries. A limitation of such data collection is that there might be some interventions that were practiced but not well documented in medical records. However, this is likely to be minimal as most information in medical records of the hospitals involved in the study is standardised for the care practices considered.

In summary, few practices of perinatal health care in the nine hospitals within the four South East Asian countries were consistent with best available evidence from Cochrane reviews and the World Health Organization Reproductive Health Library recommendations. At the individual hospitals the audit results were used to evaluate the barriers to adoption of appropriate practices and elimination of inappropriate practices followed by strategies to increase the use of evidence-based practices in perinatal health care. The recording of clinical practices within this audit was a pre-requisite to identifying problems to be addressed.

Based on our findings, the SEA-ORCHID project team developed and implemented interventions that aimed to build capacity for research conduct, synthesis and translation that would increase compliance with evidence-based clinical practice recommendations and so improve maternal and perinatal care.

\section{Acknowledgments}

The following persons and institutions participated in the SEA-ORCHID Study Group.

\section{Writing committee:}

M. Laopaiboon, P. Lumbiganon, SJ. McDonald, DJ. Henderson-Smart,

S. Green and CA. Crowther

Statistical Analyses:

M. Laopaiboon, SJ. McDonald

Project Investigators:

P. Lumbiganon, MR. Festin, JJ. Ho, M Hakimi, DJ. Henderson-Smart,

S. Green and CA. Crowther

Project Goordinator:

SJ. McDonald

Project Administrators:

M. Thomas, N. Narash

Secreterial support for paper:

M. Ewens

Data collection and entry at participating hospitals:

INDONESIA

Country Investigator: M. Hakimi

Dr Sardjito Hospital, Yogyakarta, Indonesia

Data collection: Supiyah.

Data entry: L. Amanah, D. Lukitasari and D. Astuti

Sleman District Hospital, Yogyakarta, Indonesia

Data collection: L. Amanah

Data entry: L. Amanah, D. Lukitasari and D. Astuti

MALAYSIA

Country Investigator: JJ. Ho

Ipoh Hospital, Perak, Malaysia

Data collection: SS. Cham

Data entry: SS. Cham

Universiti Sains Malaysia, Kota Bharu, Malaysia

Data collection: ET. Aw

Data entry: SS. Cham

THAILAND

Country Investigator: P. Lumbiganon

Kalasin General Hospital, Kalasin, Thailand

Data collection: B. Khianman, A. Pharprapa, J. Nachaipet

Data entry: N. Narash and R. Poombankor

Khon Kaen Regional Hospital, Khon Kaen, Thailand

Data collection: B. Saenrien, N. Srisutthikamol, S. Panikom, C.

Khunudom, S. Thipawat, S. Choonhapran, S. Nuanbuddee, P. Jarud-

phan, A. Hempira

Data entry: N. Narash and R. Poombankor

Srinagarind Khon Kaen Univeristy Hospital, Khon Kaen,

Thailand

Data collection: P. Tharnprisan, P. Sarapon, O. Ponpun

Data entry: N. Narash and R. Poombankor

THE PHILIPPINES

Country Investigator: MR. Festin

Jose Fabella Hospital, Manila, Philippines

Data collection: C. Ubaldo-Anzures and LN. Canete

Data entry: Magsipoc and JL. Festin

Philippines General Hospital (University of the Philippines), Manila, Philippines

Data collection: E. Torralba and LN. Canete

Data entry: Magsipoc and JL. Festin

AUSTRALIA

Country Investigators: D.Henderson-Smart, S. Green, CA. Crowther

Project Coordinator: SJ. McDonald.

\section{Author Contributions}

Conceived and designed the experiments: CAC. Performed the experiments: CAC. Analyzed the data: CAC. Contributed reagents/materials/ analysis tools: CAC. Wrote the paper: CAC. Interpreted the data: CAC. Involved in the development of the design of the project: CAC. 


\section{References}

1. Department of Reproductive Health and Research World Health Organization (2004) Maternal mortality in 2000: estimates developed by WHO, UNICEF and UNFPA. Geneva: WHO, [http://www.who.int/reproductive-health/publications/maternal_mortality_2000/index.html].

2. Global Health Council (2002) Making Childbirth Safer Through Promoting Evidence-Based Care. Washington, DC: Global Health Council. [http://www. globalhealth.org/sources/view.php3?id = 287].

3. Lawn J, Cousens S, Zupan J (2005) 4 million neonatal deaths: When? Where? Why?. Lancet 365(9462): 891-900.

4. Darmstadt G, Bhutta Z, Cousens S, Adam T, Walker N, et al. (2005) Evidencebased, cost-effective interventions: how many newborn babies can we save? Lancet 365(9463): 977-988.

5. Henderson-Smart D, Lumbiganon P, Festin M, Ho J, Mohammad H, et al. (2007) Optimising reproductive and child health outcomes by building evidencebased research and practice in South East Asia (SEA-ORCHID): study protocol. BMC Medical Research Methodology 7(43).

6. The WHO Reproductive Health Library (2004) Update Software. Volume 7, Oxford.

7. The Cochrane Library (2004) Issue 1. Chichester. Wiley.

8. StataCorp (2003) Stata Statistical Software: Release 8. College Station, TX: Stata Corp LP.

9. Evidence-based obstetric care in South Africa-influencing practice through the 'Better Births Initiative'. South African Medical Journal 94: 117-120.

10. Brown H, Hofmeyr GJ, Nikodem VC, Smith HJ, Garner P (2007) . Promoting childbirth companions in South Africa: A randomised pilot study. BMC Medicine 5: 7 .

11. Choices and Challenges in Changing Childbirth Research Network (2005) Routines in facility-based maternity care: evidence from the Arab World. BJOG: An International Journal of Obstetrics and Gynaecology 112(9): 1270-1276.

12. Khalil K, Elnoury A, Cherine M, Sholkamy H, Hassanein N, et al. (2005) Hospital practice versus evidence-based obstetrics: categorizing practices for normal birth in an Egyptian teaching hospital. Birth 32(4): 283-290.
13. Qian X, Smith H, Zhou L, Liang J, Garner P (2001) Evidence-based obstetrics in four hospitals in China: An observational study to explore clinical practice, women's preferences and provider's views. BMC Pregnancy and Childbirth 1(1): 1.

14. Smaill F, Hofmeyr G (2002) Antibiotic prophylaxis for cesarean section. Cochrane Database Syst Rev 2002(3): CD000933.

15. Kenyon S, Boulvain M, Neilson J (2003) Antibiotics for preterm rupture of membranes. Cochrane Database Syst Rev 2003(2): CD001058.

16. Roberts D, Dalziel S (2006) Antenatal corticosteroids for accelerating fetal lung maturation for women at risk of preterm birth. Cochrane Database Syst Rev 2006(3): CD004454

17. Hoddnet E, Gates S, Hofmeyr G, Sakala C (2007) Continuous support for women during childbirth. Cochrane Database Syst Rev 2007(3): CD003766.

18. Duley L, Gulmezoglu A, Henderson-Smart D (2003) Magnesium sulphate and other anticonvulsants for women with pre-eclampsia. Cochrane Database Syst Rev 2003(2): CD000025.

19. Duley L, Henderson-Smart D (2003) Magnesium sulphate versus diazepam for eclampsia. Cochrane Database Syst Rev 2003(4): CD000127.

20. Duley L, Henderson-Smart D (2003) Magnesium sulphate versus phenytoin for eclampsia. Cochrane Database Syst Rev 2003(4): CD000128.

21. Prendiville W, Elbourne D, McDonald S (2000) Active versus expectant management in the third stage of labour. Cochrane Database Syst Rev 2000(3): CD000007.

22. Lee C, Gong Y, Brok J, Boxall EH, Gluud C O Hepatitis B immunisation for newborn infants of hepatitis B surface antigen-positive mothers. Cochrane Database of Systematic Reviews 2006, Issue 2. Art. No.: CD004790. DOI:10.1002/ 14651858.CD004790.pub2.

23. Carroli G, Belizan J (1999) Episiotomy for vaginal birth. Cochrane Database Syst Rev 1999(3): CD000081.

24. Basevi V, Lavendar T (2000) Routine perineal shaving on admission to labour. Cochrane Database Syst Rev 2000(4): CD001236.

25. Cuervo L, Rodriguez M, Delgado M (1999) Enemas during labour. Cochrane Database Syst Rev 1999(4): CD000330. 